\title{
Effect of cilostazol on experimental diabetic neuropathy in the rat
}

\author{
M. Kihara, J. D. Schmelzer, P. A. Low \\ Neurophysiology Laboratory, Department of Neurology, Mayo Foundation, Rochester, Minnesota, USA
}

Summary Two proposed mechanisms of diabetic neuropathy are microvascular ischaemia and a reduction in Na,K-ATPase activity. We evaluated the effect of cilostazol, a drug that is both a potent phosphodiesterase inhibitor that normalizes nerve $\mathrm{Na}, \mathrm{K}$ AT-Pase and a vasodilator, on nerve blood flow (NBF) to determine whether it would improve experimental diabetic neuropathy. We examined whether epineurally applied cilostazol acted as a vasodilator on the peripheral nerve of normal and diabetic rats, and whether feeding the rats a cilostazol-supplemented diet could improve diabetic neuropathy. Cilostazol increased nerve blood flow (NBF) in a dose-dependent fashion with an $\mathrm{EC}_{50}$ of $10^{-5.74} \mathrm{~mol} / \mathrm{l}$.
Cilostazol also normalized NBF in experimental diabetic neuropathy with a $10^{-4} \mathrm{~mol} / \mathrm{l}$ local application on the sciatic nerve. In diabetic neuropathy, a cilostazol-supplemented diet improved both NBF and nerve conduction in a dose- and time-dependent fashion. Potential mechanisms of action of cilostazol on the nerve include its effect on NBF, Na, K-ATPase, and restoration of the thromboxane:prostacyclin ratio. Cilostazol may have potential in the treatment of diabetic neuropathy. [Diabetologia (1995) 38: 914 918]

Key words Diabetic neuropathy, nerve blood flow, vasodilator, sciatic nerve, cilostazol.
In experimental diabetic neuropathy (EDN), nerve blood flow (NBF) is reduced and the oxygen tension histogram is shifted into the hypoxic range [1]. Moreover, hypoxia per se, in otherwise normal nerves, will reproduce much of the lipid and electrophysiologic abnormalities of EDN [2]. Therapeutically, increasing the oxygen supply by supplementation or hyperbaric oxygenation has been shown to prevent and reverse nerve electrophysiology $[3,4]$. An alternative approach is to increase nerve blood flow by chemical

Received: 17 October 1994 and in revised form: 16 February 1995

Corresponding author: Dr. P. A. Low, Mayo Clinic, 200 First Street Southwest, Rochester, MN 55905, USA

Abbreviations: EDN, Experimental diabetic neuropathy; NBF, nerve blood flow; STZ, streptozotocin; NRC, control rats receiving normal diet; $\mathrm{NRH}$, control rats receiving a high $(0.1 \%)$ cilostazol diet; CSH, STZ rats receiving high $(0.1 \%)$ cilostazol diet; CSL, STZ rats receiving low $(0.03 \%)$ cilostazol diet; CV, conduction velocity. sympathectomy [5], vasodilators, such as $\alpha$-antagonists [5], or eicosanoids [6]. Cilostazol (6-[4-(1-cyclohexyl-1H-tetrazol-5-yl)butoxy]-3,4-dihydro-2(1H)quinolinone) is a potent phosphodiesterase inhibitor $[6,7]$. It blocks platelet aggregation and is a vasodilator of cerebral and femoral arteries $[8,9]$. Its effects on peripheral nerves are unknown. We tested the hypothesis that cilostazol may be a peripheral nerve vasodilator, and that it might be effective in improving reduced NBF in EDN. We evaluated both the topical application (to elucidate its mode of action, and establish a dose-response relationship), and oral feeding in the prevention of NBF and conduction abnormalities in EDN.

\section{Materials and methods}

Animals. We used male Sprague Dawley rats with initial weights of about $250 \mathrm{~g}$. These animals were housed in cages with plastic floors covered with wood shavings and were fed 
Purina rat chow (no. 5001; Purina, St. Louis, MO, USA) with an unrestricted supply of water.

Experimental diabetes was induced with streptozotocin (STZ), administered by intraperitoneal injection. STZ was dissolved in $0.05 \mathrm{~mol} / \mathrm{l}$ sodium citrate at $\mathrm{pH} 4.5(65 \mathrm{mg} / \mathrm{ml}$; dose $=1.32 \mathrm{ml} / \mathrm{kg}$ ). Rats were presumed diabetic if their fasting blood glucose level exceeded $16.7 \mathrm{mmol} / 17$ days after injection of STZ and remained greater than $16.7 \mathrm{mmol} / \mathrm{l}$ throughout the study.

We evaluated the effects of the epineural application of cilostazol on sciatic NBF in normal rats in order to determine whether it had a vasodilator action, and if it could normalize the reduced NBF of EDN and to obtain a dose-effect relationship. We also evaluated the effect of oral cilostazol supplementation in reversing STZ-induced EDN. Cilostazol was provided as a gift by Otsuka Pharmaceutical Co., Ltd., Tokoshima, Japan.

Local application. We used a previously described nerve preparation [10]. In brief, rats were anaesthetized with an i.p. injection of barbiturate (pentobarbital; $100 \mathrm{mg} / \mathrm{kg}$ ), paralysed with curare ( $2 \mathrm{U}$ i.v. and $6 \mathrm{U}$ i.p.), and ventilated using a rodent respirator supplying a mixture of nitrogen and oxygen to maintain arterial blood gases within the physiological range $(\mathrm{pH}$ $7.4 \pm 0.5 ; \mathrm{pO}_{2} 110 \pm 20 ; \mathrm{pCO}_{2} 40 \pm 5 \mathrm{mmHg}$ ).

The nerve preparation has been especially modified for the study of vasoactive agents on peripheral nerve [10]. A length of left sciatic nerve not exceeding $2 \mathrm{~cm}$ was exposed and a pool was formed by the surrounding muscle and skin. The pool was filled with mammalian Ringer's solution and the temperature was maintained at $33.5 \pm 0.1^{\circ} \mathrm{C}$ using a servo-controlled infrared heat lamp. Mammalian Ringer's solution was composed of: (in mmol/I) Na 145, K 3.5, Ca 2, glucose 6 and Hepes buffer 2 and adjusted to $\mathrm{pH} 7.4$. Rectal temperature was maintained between 36.5 and $37.5^{\circ} \mathrm{C}$ by infrared lamp. The nerve was superfused with solutions of known concentrations of cilostazol. The effects of cilostazol at concentrations of $10^{-9}, 10^{-7}, 10^{-6}$, $10^{-5}$, and $10^{-4} \mathrm{~mol} / \mathrm{l}$ were examined in a normal nerve and at concentration of $10^{-4} \mathrm{~mol} / \mathrm{l}$ in a diabetic nerve. The rat was killed with an overdose of pentobarbital at the end of the study.

Cilostazol supplementation study. We separated rats into five groups. Each group received their respective diets for a total of 4 weeks.

(1) Controls receiving normal diet (NRC), $n=6$

(2) Controls receiving a high $(0.1 \%)$ cilostazol diet for 4 weeks

(NRH), $n=7$

(3) STZ receiving normal diet (STZ), $n=5$

(4) STZ receiving high ( $0.1 \%$ ) cilostazol diet (CSH), $n=6$

(5) STZ receiving low (0.03\%) cilostazol diet (CSL), $n=6$

To ensure that the cilostazol-supplemented rats were neuropathic, we performed nerve conduction studies in STZ rats before cilostazol feeding and only those rats with motor conduction velocities in sciatic-tibial nerve of $30 \mathrm{~m} / \mathrm{s}$ or lower were used.

Measurement of NBF. NBF was measured by microelectrode hydrogen polarography (tip size $2-5 \mu \mathrm{m}$ ) [10]. The signal was input into a computer via an analogue-to-digital converter (Colbourn Instruments, Lehigh Valley, Pa., USA) for simultaneous display (Labtech Notebook Laboratory Technologies Corp., Cambridge, Mass., USA) and storage (Lotus 123, Lotus Development Corp., Cambridge, Mass., USA). A curve was fitted to the data using a non-linear regression program based on the Marquardt algorithm [11]. Records were taken from the centre of the fascicle. Blood pressure and heart rate were continuously monitored.
Electrophysiology. In the electrophysiological studies we used techniques that are standard for our laboratory. Motor conduction velocities and the amplitude of the compound muscle action potential were measured in the sciatic-tibial and caudal nerves [12]. Sensory conduction velocities and nerve action potential amplitude were measured in the caudal nerve [13]. Fine stainless steel near-nerve stimulating and recording electrodes were employed. Recordings were made at $35^{\circ} \mathrm{C}$, amplified 1000 times, stored on computer discs, and analysed off-line using a Nicolet 4094 digital oscilloscope (Madison, WI, USA) with associated stimulators and stimulus isolation units.

Blood glucose level. Blood glucose level was determined at 4 weeks using glucostix reagent strips and a glucose meter (Ames Glucometer II, model 5550, Elkhart, IN, USA). Rats were presumed diabetic if their blood glucose level was greater than $19.6 \mathrm{mmol} / \mathrm{l}$.

\section{Statistical analysis}

Statistical analysis was performed on a $486 \mathrm{PC}$ computer system with Systat for Windows (Systat Inc., Evanston, Ill., USA) statistical program. Curve fitting to derive EC50 and asymptote were carried out using the Graphpad curve-fitting and plotting program (ISI Software, Philadelphia, Pa., USA). Group data were compared using two-tailed unpaired $t$-test. Values are expressed as mean \pm SEM and significance was accepted when $p<0.05$.

\section{Results}

Blood glucose level. The blood glucose values (mean $\pm \mathrm{SEM}$ ), at time of killing were as follows: NRC, $5.1 \pm 0.2$; NRH, 5.2 \pm 0.3 ; STZ, greater than 22.5; CSH, greater than 22.5; CSL, greater than $22.5 \mathrm{mmol} / 1$. The diabetic groups all had significantly higher blood glucose levels $(p<0.001)$ than controls and controls with high cilostazol diet.

Body weight. Body weight values at time of killing were as follows: NRC, 412.6 $\pm 11.3 ; \mathrm{NRH}, 432.0 \pm$

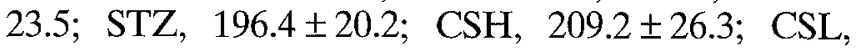
$176.4 \pm 22.1 \mathrm{~g}$. The diabetic groups all had significantly lower weights $(p<0.001)$ than controls and controls with high cilostazol diet.

\section{Dose-response study: epineurially applied cilostazol}

(1) Normal nerve: A dose-response study was performed on the sciatic nerve of six control rats. Cilostazol was superfused at concentrations of $10^{-9}, 10^{-7}$, $10^{-6}, 10^{-5}$, and $10^{-4} \mathrm{~mol} / \mathrm{l}$. Cilostazol increased NBF in a dose-dependent fashion (Fig. 1). Resting NBF was $14.3 \pm 1.0 \mathrm{ml} \cdot 100 \mathrm{~g}^{-1} \cdot \mathrm{min}^{-1}$. A dose of $10-9 \mathrm{ap}-$ peared to be the threshold dose and an increase of $52.7 \pm 16.9 \%$ occurred with $10^{-4} \mathrm{~mol} / \mathrm{l}$. The $\mathrm{EC}_{50}$ was $10^{-5.74} \mathrm{~mol} / \mathrm{l}$. Arterial blood pressure remained constant throughout the experiments. Corresponding 


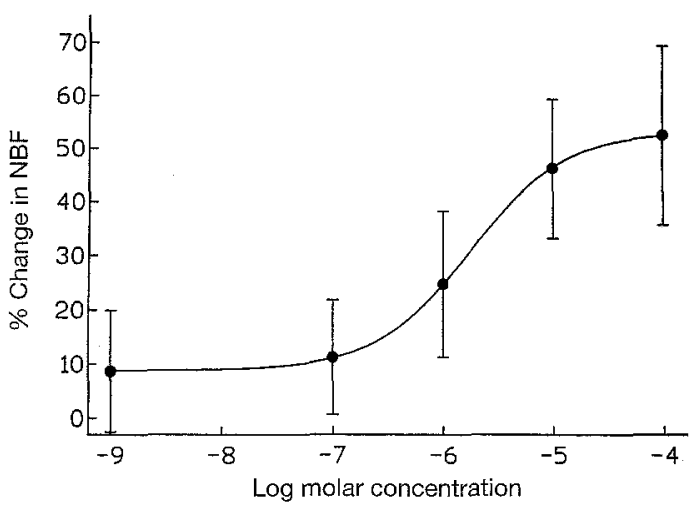

Fig. 1. Dose-response study relating nerve blood flow, expressed as percent change against log-molar concentration of epineurally applied cilostazol. Bars represent \pm SEM

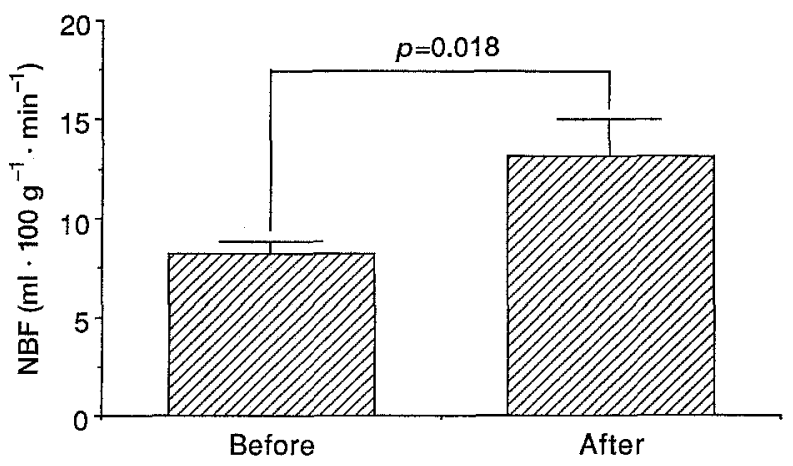

Fig. 2. Effect of epineurally applied cilostazol on sciatic nerve blood flow of experimental diabetic neuropathy

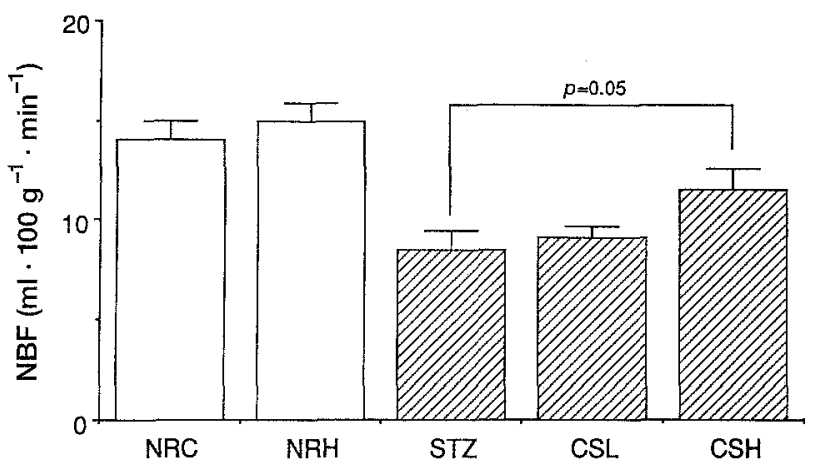

Fig. 3. The effect of oral supplementation of cilostazol for 4 weeks on sciatic nerve blood flow of control (NRC and $\mathrm{NRH}$ ) and diabetic (STZ) rats in low (CSL) and high (CSH) concentration

values for nerve vascular resistance in $\mathrm{mmHg} \cdot \mathrm{ml}^{-1}$. $\min ^{-1} \cdot 100 \mathrm{~g}^{-1}$ were $15.0+0.6,9.6+0.6,9.8+0.8$, $7.5+0.5$, and $6.9+0.7$ for $10^{-9}, 10^{-7}, 10^{-6}, 10^{-5}$ and $10^{-4}$ $\mathrm{mol} / 1$, respectively.

(2) Diabetic neuropathy: The diabetic state resulted in a significant reduction $(p<0.05)$ in NBF $(8.2 \pm 0.6$ $\left.\mathrm{ml} \cdot 100 \mathrm{~g}^{-1} \cdot \mathrm{min}^{-1}\right)$ when compared with controls $\left(14.3 \pm 1.0 \mathrm{ml} \cdot 100 \mathrm{~g}^{-1} \cdot \mathrm{min}^{-1}\right)$. The reduction in flow

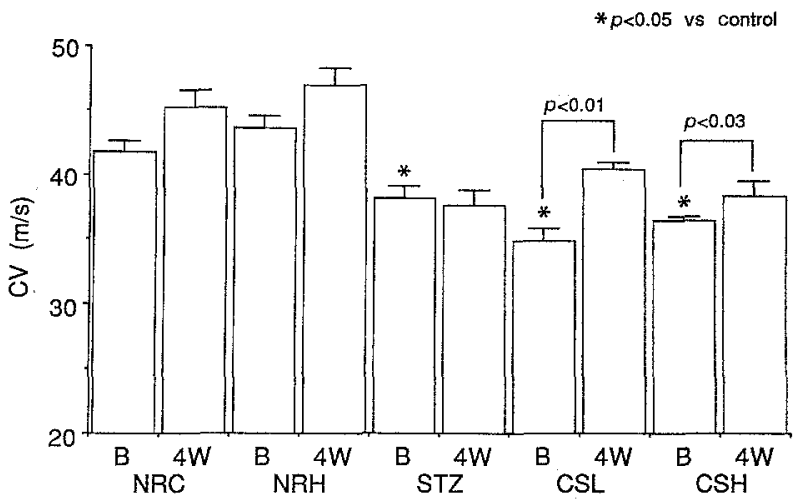

Fig.4. The effect of oral supplementation of cilostazol for 4 weeks $(4 \mathrm{~W})$ on sciatic-tibial nerve conduction velocity (CV) on controls (NRC and NRH), diabetics (STZ) in low (CSL) and high (CSH) concentrations. $\mathrm{B}$ and $4 \mathrm{~W}$ refer to values before and 4 weeks after supplementation

was associated with a significant increase in microvascular nerve resistance. Both NBF and vascular resistance (not shown) were normalized with local application of $10^{-4} \mathrm{~mol} / \mathrm{l}$ cilostazol (Fig. 2 ).

\section{Oral supplementation of cilostazol on experimental diabetic neuropathy}

Following 4 weeks of dietary manipulation, NBF was measured in the left sciatic nerve. This was followed by electrophysiological studies on the same animals. Blood was also drawn for determination of blood glucose level.

(1) Nerve blood flow: The STZ group had a significant reduction in NBF when compared with controls $(p<0.05)$. Cilostazol supplementation had no effect on control nerves, while its effect on diabetic nerves consisted of a modest non-significant improvement at low doses and improved NBF in the CSH group (STZ vs CSH, $p<0.05$; NRC vs CSH, $p>0.05$; Fig. 3 ).

(2) Electrophysiology: Sciatic-tibial nerve conduction velocity was significantly reduced in the diabetic animals (STZ vs NRC, $p<0.05$ ). Cilostazol treatment improved nerve conduction velocity in both CSL $(p<0.01)$ and $\mathrm{CSH}(p<0.03)$ (Fig. 4$)$.

Caudal conduction velocity was reduced in the STZ group and became more abnormal with increasing duration of diabetes. Cilostazol treatment improved conduction velocity in a dose-dependent manner, reaching statistical significance in the $\mathrm{CSH}$ group $(p<0.03)$ (Fig. 5). 


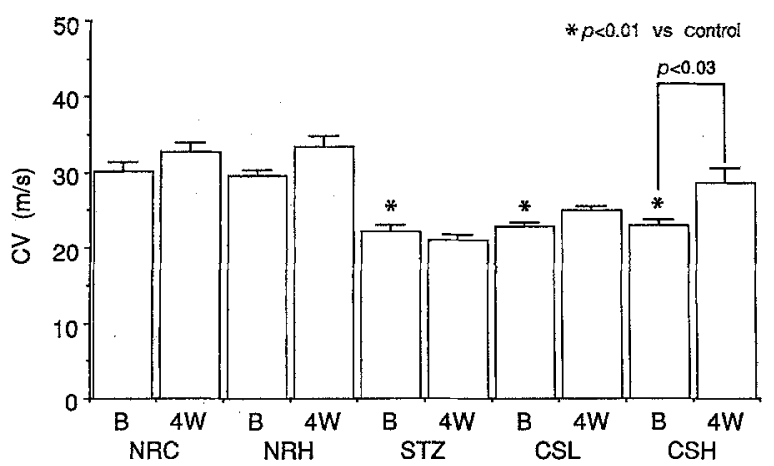

Fig. 5. The effect of oral supplementation of cilostazol for 4 weeks $(4 \mathrm{~W})$ on caudal nerve conduction velocity (CV) on controls (NRC and NRH), diabetics (STZ) in low (CSL) and high $(\mathrm{CSH})$ concentrations. $\mathrm{B}$ and $4 \mathrm{~W}$ refer to values before and 4 weeks after supplementation

\section{Discussion}

The main findings of this study are that cilostazol is a potent vasodilator of rat peripheral nerves, and that it will acutely normalize the reduction in NBF in EDN. Oral feeding will improve both the NBF and conduction slowing of EDN.

We used a combination of microelectrode polarography and topical superfusion to test this agent. This method has two major advantages. The first is the avoidance of a systemic effect, an important property, since the systemic effect of vasoactive agents tends to overwhelm the lesser effects on the nerve microvascular bed. The second advantage of our preparation is the ability of topically applied agents to rapidly bind to epineural receptors, vascular smooth muscle and, because of a lack of a blood-nerve barrier, to endothelial receptors.

Cilostazol has several properties potentially beneficial to diabetic neuropathy. Vasodilator effects of cilostazol have been shown in the dog where the percent dilatation using cilostazol $(100 \mathrm{ml} / \mathrm{min})$ in the femoral artery, vertebral artery, internal carotid artery, and renal artery was $138.0 \pm 19.5,74.4 \pm 10.5$, $41.1 \pm 6.2$ and $7.1 \pm 0.8 \%$, respectively [8]. Our results indicate that cilostazol has a direct vasodilator effect on epineurial arterioles of peripheral nerve producing similar effects to those seen in the vertebral artery. The magnitude of vasodilatation is similar to that achievable with phentolamine [14], guanethidine [5, $14]$, with the vasodilator peptides such as substance $P$ and calcitonin gene related peptide [15], and vasodilator eicosanoids (Kihara and Low, unpublished data). The $\mathrm{EC}_{50}$ of $10^{-5.7} \mathrm{~mol} / \mathrm{l}$ is similar to that of prostacyclin $\left(10^{5.1} \mathrm{~mol} / \mathrm{l}\right)$, prostaglandin $\mathrm{E}_{1}\left(10^{-6.7} \mathrm{~mol} / \mathrm{l}\right)$, and prostaglandin $\mathrm{F}_{2 \alpha}\left(10^{-5.8} \mathrm{~mol} / \mathrm{l}\right)$.

From calculations of food consumption, rats receiving food with concentrations of $0.03 \%$ and $0.1 \%$ cilostazol receive the equivalent of 58.6 and 195.9 $\mathrm{mg} \cdot \mathrm{kg}^{-1} \cdot \mathrm{day}^{-1}$. Determinations of plasma cilostazol using the method of Akiyama et al. [7] on five normal rats fed $0.1 \%$ and two groups of five diabetic rats fed $0.03 \%$ and $0.1 \%$ cilostazol, respectively yielded values of $107 \pm 12,370 \pm 131$ and $522 \pm 151$ ng/ml, respectively (Dr. Y.Kimura, Otsuka Pharmaceutical Co., Ltd, Tokushima, Japan; personal communication). These levels are well within the range found in human subjects fed a single $100 \mathrm{mg}$ dose. Cilostazol in humans was well tolerated and was found to be rapidly absorbed, reaching peak plasma levels of $764 \mathrm{ng} / \mathrm{ml}$ at $3 \mathrm{~h}$ with an elimination half-life of $2.2 \mathrm{~h}$ ( $\alpha$-phase) and $18.0 \mathrm{~h}$ ( $\beta$-phase) [7].

Cilostazol could improve peripheral nerve function by means of several mechanisms. First, conduction slowing has been ascribed to reduced nerve $\mathrm{Na}, \mathrm{K}$-ATPase activity [16]. Cilostazol has been shown to normalize this deficiency in STZ-diabetic nerves [17]. Cilostazol did not improve blood glucose levels; thus its beneficial effects are clearly unrelated to correction of hyperglycaemia. Shindo et al. [17] has previously reported electrophysiologic improvement without any correction of blood glucose levels or of myo-inositol in EDN. Our study is the first to demonstrate that cilostazol has a direct effect on NBF, suggesting that an alternative or additional mechanism for improving nerve electrophysiology might be through improvement in endoneurial perfusion. A second action relevant to diabetes is its potent inhibition of cGMP-inhibited, cAMP-selected phosphodiesterase (type III) [8] with an $\left(\mathrm{IC}_{50}\right)$ of $0.19 \mu \mathrm{mol} / \mathrm{l}$. A third action, of particular relevance to the diabetic peripheral nerve, is its ability to lower thromboxane $\mathrm{B}_{2}$ without altering 6-keto prostaglandin $F_{1 \alpha}$, the stable metabolite of prostacyclin. There was restoration of the ratio toward normal both in an experimental preparation [18] and in human subjects [19]. The thromboxane $\mathrm{B}_{2}$ : prostacyclin ratio is increased in EDN [20]. The demonstration that oral administration will improve the NBF alterations and electrophysiologic abnormalities in EDN are especially important in suggesting that ingestion of the drug is potentially efficacious in diabetic neuropathy. Controlled clinical studies in human diabetic neuropathy are needed. EDN is very important in creating a model for early neuropathic alterations, but the chronic progressive diabetic changes in human nerve tissue probably involves additional mechanisms.

Acknowledgements. Supported in part by grants from NINCDS (RO1 NS22352 and NS30534), MDA, and Mayo Funds. Dr. Low is the recipient of a Jacob Javits Neuroscience Investigator Award.

\section{References}

1. Tuck RR, Schmelzer JD, Low PA (1984) Endoneurial blood flow and oxygen tension in the sciatic nerves of rats with experimental diabetic neuropathy. Brain 107: 935-950 
2. Yao JK, Low PA (1986) Improvement of endoneurial lipid abnormalities in experimental diabetic neuropathy by oxygen modification. Brain Res 362: 362-365

3. Low PA, Schmelzer JD, Ward KK, Curran GL, Poduslo JF (1988) Effect of hyperbaric oxygenation on normal and chronic streptozotocin diabetic peripheral nerves. Exp Neurol 99: 201-212

4. Low PA, Tuck RR, Dyck PJ, Schmelzer JD, Yao JK (1984) Prevention of some electrophysiologic and biochemical abnormalities with oxygen supplementation in experimental diabetic neuropathy. Proc Natl Acad Sci U S A 81: 68946898

5. Cameron NE, Cotter MA, Low PA (1991) Nerve blood flow in early experimental diabetes in rats: relation to conduction deficits. Am J Physiol 261: E1-E8

6. Sonobe M, Yasuda H, Hisanaga T et al. (1991) Amelioration of nerve $\mathrm{Na}(+)-\mathrm{K}(+)$-ATPase activity independently of myo-inositol level by PGE1 analogue OP1206.alpha-CD in streptozocin-induced diabetic rats. Diabetes 40: 726-730

7. Akiyama H, Kudo S, Odomi M, Shimizu T (1985) High-performance liquid chromatographic procedure for the determination of a new antithrombotic and vasodilating agent, cilostazol, in human plasma. J Chromatogr 338: 456-459

8. Okuda Y, Kimura Y, Yamashita K (1993) Cilostazol. Cardiovasc Drug Rev 11: 451-465

9. Kawamura K, Watanabe K, Kimura Y (1985) Effect of cilostazol, a new antithrombotic drug, on cerebral circulation. Arzneimittelforschung 35: 1149-1154

10. Kihara M, Low PA (1990) Regulation of rat nerve blood flow: role of epineurial alpha-receptors. J Physiol 422: 145-152

11. Marquardt DW (1963) An algorithm for least-squares estimation of nonlinear parameters. J Soc Indust Appl Math 11: $431-441$
12. Low PA, Schmelzer JD (1983) Peripheral nerve conduction studies in galactose-poisoned rats. Demonstration of increased resistance to ischemic conduction associated with endoneurial edema due to sugar alcohol accumulation. J Neurol Sci 59: 415-421

13. Low PA, Ward K, Schmelzer JD, Brimijoin S (1985) Ischemic conduction failure and energy metabolism in experimental diabetic neuropathy. Am J Physiol 248: E457$\mathrm{E} 462$

14. Zochodne DW, Huang ZX, Ward KK, Low PA (1990) Guanethidine- induced adrenergic sympathectomy augments endoneurial perfusion and lowers endoneurial microvascular resistance. Brain Res 519: 112-117

15. Zochodne DW, Ho LT (1991) Influence of perivascular peptides on endoneurial blood flow and microvascular resistance in the sciatic nerve of the rat. J Physiol 444: 615630

16. Greene DA, Lattimer SA (1983) Impaired rat sciatic nerve sodium-potassium adenosine triphosphatase in acute streptozocin diabetes and its correction by dietary myo-inositol supplementation. J Clin Invest 72: 1058-1063

17. Shindo H, Tawata M, Onaya T (1993) Cyclic adenosine $3^{\prime}, 5^{\prime}$-monophosphate enhances sodium, potassium-adenosine triphosphatase activity in the sciatic nerve of streptozotocin-induced diabetic rats. Endocrinology 132: 510516

18. Igawa T, Tani T, Chijiwa Tet al. (1990) Potentiation of antiplatelet aggregating activity of cilostazol with vascular endothelial cells. Thrombosis 57: 617-633

19. Nakagawa Y, Onuki Y, Orino H (1986) Effect of cilostazol (10P-13013) on arachidonic acid metabolism. Jpn Pharmacol Ther 14: 6319-6324

20. Ward KK, Low PA, Schmelzer JD, Zochodne DW (1989) Prostacyclin and noradrenaline in peripheral nerve of chronic experimental diabetes in rats. Brain 112: 197-208 